\title{
Kinetic and hydrodynamic regimes in multi-particle-collision dynamics of a one-dimensional fluid with thermal walls
}

\author{
Stefano Lepri $\odot,{ }^{1,2}$ Guido Ciraolo, ${ }^{3}$ Pierfrancesco Di Cintio $\odot,{ }^{2,4}$ Jamie Gunn, ${ }^{3}$ and Roberto Livi ${ }^{1,2,4}$ \\ ${ }^{1}$ Consiglio Nazionale delle Ricerche, Istituto dei Sistemi Complessi via Madonna del piano 10, I-50019 Sesto Fiorentino, Italy \\ ${ }^{2}$ Istituto Nazionale di Fisica Nucleare, Sezione di Firenze, via Giovanni Sansone 1, I-50019 Sesto Fiorentino, Italy \\ ${ }^{3}$ CEA, IRFM, F-13108 Saint-Paul-lez-Durance, France \\ ${ }^{4}$ Dipartimento di Fisica e Astronomia and CSDC, Universitá di Firenze, via Giovanni Sansone 1, I-50019 Sesto Fiorentino, Italy
}

(Received 6 October 2020; accepted 5 January 2021; published 5 March 2021)

\begin{abstract}
We study the nonequilibrium steady states of a one-dimensional fluid in a finite-space region of length $L$. Particles interact among themselves by multi-particle collisions and are in contact with two thermal-wall heat reservoirs, located at the boundaries of the region. After an initial ballistic regime, we find a crossover from a normal (kinetic) transport regime to an anomalous (hydrodynamic) one, above a characteristic size $L_{*}$. We argue that $L_{*}$ is proportional to the cube of the collision time among particles. Motivated by the physics of emissive divertors in fusion plasma, we consider the more general case of thermal walls injecting particles with given average (nonthermal) velocity. For fast and relatively cold particles, short systems fail to establish local equilibrium and display non-Maxwellian distributions of velocities.
\end{abstract}

DOI: 10.1103/PhysRevResearch.3.013207

\section{INTRODUCTION}

Statistical systems driven away from equilibrium by external agents, such as concentration and/or thermal gradients, forced flows, etc., are ubiquitous in nature and in technological applications. Especially in far-from-equilibrium regimes, simulation of model systems is a basic tool to gain insight into the fundamental properties of the system under study. Molecular dynamics is the most natural approach, but methods based on effective stochastic processes may be a computationally convenient alternative. In particular, the multi-particle-collision (MPC) dynamics can be considered as a mesoscale simulation method where particles undergo stochastic collisions. The implementation was originally proposed by Malevanets and Kapral [1] and Kapral [2] and consists of two distinct stages: a free-streaming and a collision one. Collisions occur at fixed discrete time intervals, and space is discretized into cells that define the collision range. The MPC dynamics is a useful tool not only to investigate concrete systems such as polymers in solution, colloidal fluids, etc., but also to address fundamental problems in statistical physics and, in particular, the effect of external sources.

In this paper, we investigate the transport property of a one-dimensional gas driven off equilibrium by different reservoirs, modeled as thermal walls. This general setup has been considered very often in the literature $[3,4]$ with several possible applications [5]. For the discussion that follows, it is

Published by the American Physical Society under the terms of the Creative Commons Attribution 4.0 International license. Further distribution of this work must maintain attribution to the author(s) and the published article's title, journal citation, and DOI. worth recalling that the prediction of nonlinear fluctuating hydrodynamics [6,7] for one-dimensional (1D) systems, constrained by three global conservation laws, is that transport is generically anomalous and belongs to the Kardar-ParisiZhang (KPZ) dynamical universality class. This is confirmed by other approaches including hydrodynamics [8,9], kinetic theory [10-12], and many numerical simulations [13-15]. However, for finite systems some deviations are found, and it is thus of interest to clarify which are the different transport regimes (see, e.g., Refs. [16,17] for an up-to-date account). In this framework, simple kinetic models are a valuable testbed.

Besides the general theoretical motivations, our interest is also in application of the technique in plasma physics $[18,19]$. We wish to investigate the MPC method as a tool to study nonequilibrium properties of fusion plasma which are subject to large temperature gradients. This is a relevant issue in the modeling of plasma transport in the direction parallel to the magnetic field at the edge of magnetic fusion devices where hot plasma regions are connected to a wall component. In this case a significant temperature gradient along the field line sets in between the hot region, which acts as a heat source, and the colder plasma region at the wall, which acts as a sink. Deviations from the classical Fourier law have been observed (see, for example, Refs. [20,21] and references therein) together with transitions from strongly collisional to collisionless regimes, exhibiting different heat conduction features. We want to point out that accounting for these kinetic effects by a fluid model of heat transport is of primary importance for implementing realistic and efficient hydrodynamic simulations. In this perspective, what is contained in this paper can be considered as a worthwhile evolution along the line of simplified 1D fluid models, already adopted for studying thermal transport in plasmas [22-24]. For the sake of brevity, we adopt for such a model the label 1D1V used sometimes in 
the literature, meaning that we consider particles constrained on the line with one velocity component along it.

Besides this, in the edge plasma of magnetic fusion devices, several phenomena, such as, for example, neutral beam injection, are related to the injection of hot and/or cold plasma particles. We refer, for example, to hot sources caused by external heating or to particular regimes with emissive divertor targets on the wall due to the high temperatures associated with steady-state loads, as expected, for example, on next-step large fusion devices [25,26]. Here, we will thus attempt to model this situation, by generalizing the usual thermal-wall method to include the possibility of injecting particles with an average nonthermal velocity. To our knowledge, this case has not been treated in the statistical physics literature, and it has thus an interest by itself.

The outline is as follows. In Sec. II we recall the definition of the fluid dynamics and the thermal walls. Section III discusses the crossover from the so-called kinetic (diffusive) regime to the anomalous, hydrodynamic one. The effect of general thermal walls is illustrated in Sec. IV with reference to the specific case of shifted Maxwell-Boltzmann distributions.

\section{1D1V MPC WITH THERMAL WALLS}

For a detailed description of the MPC simulation scheme we refer the reader to Refs. $[18,19]$. Here, we just summarize the basic ingredients. We consider an ensemble of $N$ point particles with equal masses $m$ located in a finite-space region, $[0, L]$, partitioned into $N_{c}$ (fixed) cells of size $a, L=N_{c} a$. The density of particles, $d=N / L$, is fixed, while all physical quantities, without prejudice of generality, are set to dimensionless units, namely, $a=1, m=1$, and (for the isolated system) $E_{c}=1$, the latter denoting the total kinetic energy of the fluid of particles.

The MPC is performed at regular time steps, separated by a constant time interval of duration $\delta t$ : The velocity of the $j$ th particle in the $i$ th cell is changed according to the update rule $v_{j, \text { old }} \rightarrow v_{j \text {, new }}=a_{i} w_{j}+b_{i}$, where $w_{j}$ is randomly sampled by a thermal distribution at the cell temperature $T_{i}$, while $a_{i}$ and $b_{i}$ are cell-dependent parameters, determined by the condition of total momentum and total energy conservation in the cell [18]. After the collision, all particles freely propagate, i.e.,

$$
x_{j}(t+\delta t)=x_{j}(t)+v_{j}(t) \delta t,
$$

and they may move to a new cell.

The nonequilibrium state is imposed by two thermal walls [27] acting at the edges of the space region: Any particle crossing the boundaries of the interval $[0, L]$ is reinjected in the opposite direction with a random velocity extracted form the Maxwell-Boltzmann distributions

$$
p_{0}(v)=\frac{v}{T_{0}} e^{-v^{2} / 2 T_{0}}, \quad p_{L}(v)=-\frac{v}{T_{L}} e^{-v^{2} / 2 T_{L}} .
$$

Reinjected particles propagate with their new velocities for the remaining time up to the next collision. Since arbitrarily large values of $v$ are admitted, a particle may reach the opposite boundary during this time, although such a process, in practice, becomes extremely unlikely, i.e., negligible, for sufficiently large values of $L$, considering that in the adopted dimensionless units the typical value of $v$ is $\mathcal{O}(1)$.

The heat fluxes at the two boundaries, $Q_{0}$ and $Q_{L}$, respectively, are computed as the average kinetic energy exchanged by particles interacting with the walls $[3,4]$. The relaxation of the fluid to a steady state, i.e., $Q_{0} \approx-Q_{L}=Q$, is monitored by controlling the convergence of the running time averages of the fluxes. The thermal conductivity is defined as $K=Q /(\Delta T / L)$, where $\Delta T=T_{0}-T_{L}$. Typically, in order to speed up the relaxation to a steady state, the initial velocities of the particles in cell $i$ are randomly extracted from a Maxwell-Boltzmann distribution at temperature $T_{i}=$ $T_{L}+\left(T_{0}-T_{L}\right) i / L$, i.e., according to the linear temperature profile compatible with Fourier's law.

The relevant kinetic parameters of the MPC protocol are the particle mean free path $\ell$, which, in a uniform system, is proportional to the thermal velocity of the fluid $v_{T}=\sqrt{T}$, according to the formula $\ell \sim v_{T} \delta t$, and the thermal conductivity, which is expected to be proportional to the typical kinetic energy of the fluid, according to the formula $K=$ $C v_{T} \ell \sim v_{T}^{2} \delta t, C$ being the specific heat at constant volume.

We conclude this section by making the reader aware of the main advantage of MPC simulations, with respect to those typically performed in nonlinear lattice models. The possibility of observing crossover between different transport regimes in the latter class of models depends on the fine-tuning of various simulation parameters, e.g., energy density and coupling with the thermal reservoirs. These features are strongly model dependent, because the crossover effects are ruled by the lifetime of typical nonlinear excitations, influencing the stationary transport process. As we show in what follows, in MPC simulations one can control different regimes essentially by a single natural parameter, the collision time $\delta t$, which is straightforwardly related to the mean free path of the fluid particles, weak and strong collisional regimes corresponding to large and small values of $\delta t$, respectively.

\section{FROM KINETIC TO HYDRODYNAMIC REGIMES}

The kind of stationary heat transport phenomenon one is dealing with is characterized by the scaling of $Q$ with the system size $L$. Diffusive transport, i.e., Fourier's law, yields $Q \propto L^{-1}$. For the 1D1V MPC, based on general theoretical arguments [6,7] we expect that transport would be anomalous and belonging to the KPZ universality class, as indicated by equilibrium correlation functions [18]. In the nonequilibrium setup used in this paper this would imply that

$$
Q \propto L^{-2 / 3}, \quad L \rightarrow \infty .
$$

However, this asymptotic anomalous regime may be eventually attained going through a crossover from a standard kinetic, i.e., diffusive, regime [17,28,29]. This scenario can be rationalized by assuming that the flux is made of two contributions

$$
Q=Q_{N}+Q_{A},
$$

where $Q_{N}$ is the "normal" part of the flux that can be written as

$$
Q_{N}=\frac{q}{r+L / \ell}
$$


(with $q$ and $r$ being suitable parameters), while $Q_{A}$ is the anomalous part that, for large enough values of $L$, should scale as

$$
Q_{A} \sim\left(\frac{b}{L}\right)^{\frac{2}{3}},
$$

where $b \sim \mathcal{O}(1)$ is a characteristic length [17]. Formula (5) is suggested by kinetic theory to account for the crossover from the initial ballistic regime to a diffusive one (see, e.g., Ref. [30] and Sec. 3.4 in Ref. [3]). It implies that up to system lengths of the order of the mean free path $L \sim \ell$, transport is essentially ballistic, with a flux independent of $L$, while for $L \gg \ell, Q_{N} \sim \frac{\ell}{L}$.

According to the above formulas, for large enough $L$ one can define a crossover length $L_{*}$ from the normal to the anomalous regime when $Q_{N} \approx Q_{A}$, i.e.,

$$
L_{*} \sim\left(\frac{\ell^{3}}{b^{2}}\right) \sim v_{T}^{3} \delta t^{3} .
$$

Altogether, upon increasing $L$ at fixed $\ell$, one should see a first ballistic regime followed by a kinetic (diffusive) one until eventually the asymptotic hydrodynamic regime is attained. It should be, however, remarked that the different regimes are observable only provided that the relevant length scales are widely separated, in such a way that the range of scales between the ballistic and anomalous regimes is large enough, i.e., $\ell \ll L_{*}$.

The numerical results reported in Fig. 1(a) provide a clear confirmation of this scenario. The energy flux $Q(L)$ displays a crossover from diffusive to anomalous scaling upon decreasing the collision time $\delta t$. In Fig. 1(b) we also show that for low collisionality and small $L$, the thermal conductivity $K$, obtained for different values of $\delta t$, tends to collapse onto a single curve after rescaling $K$ and $L$ by $\delta t$, or, equivalently, by $\ell$. This confirms that in the kinetic regime, $\ell$ is the only relevant scale as expected.

A more refined analysis of the data, supporting ansatz (4), can be obtained by estimating the two contributions in Eq. (4). This is accomplished by fitting rescaled conductivity data with the largest $\delta t$ via the functional form $\frac{A L}{B+L}$ suggested by Eq. (5), with $A$ and $B$ being fitting parameters.

As seen in Fig. 1(b) (dot-dashed line) the fitting is very accurate, meaning that the anomalous contribution to the conductivity is negligible over the considered length range. From the fitting function, one can obtain straightforwardly the best estimate of $Q_{N}$ and thus the anomalous part as $Q_{A}=Q-Q_{N}$. As shown in Fig. 2, a power-law fit of $Q_{A}$ indicates excellent agreement with the expected scaling [Eq. (3)] on a remarkably large range of about 3 decades in $L$. To our knowledge, this is one of the most solid numerical confirmations of the predictions of fluctuating hydrodynamics. We also note that the characteristic length $b$ is found to be independent of the collision parameter.

The difference between the two regimes can be further appreciated also in the temperature and density profiles computed as averages of $v_{i}^{2}$ and of the number of particles in each one of the $N_{c}$ cells. In order to deal properly with the large- $L$ limit, we plot the temperature and the density profiles, $T(x)$ and $n(x)$, respectively, as a function of rescaled variable
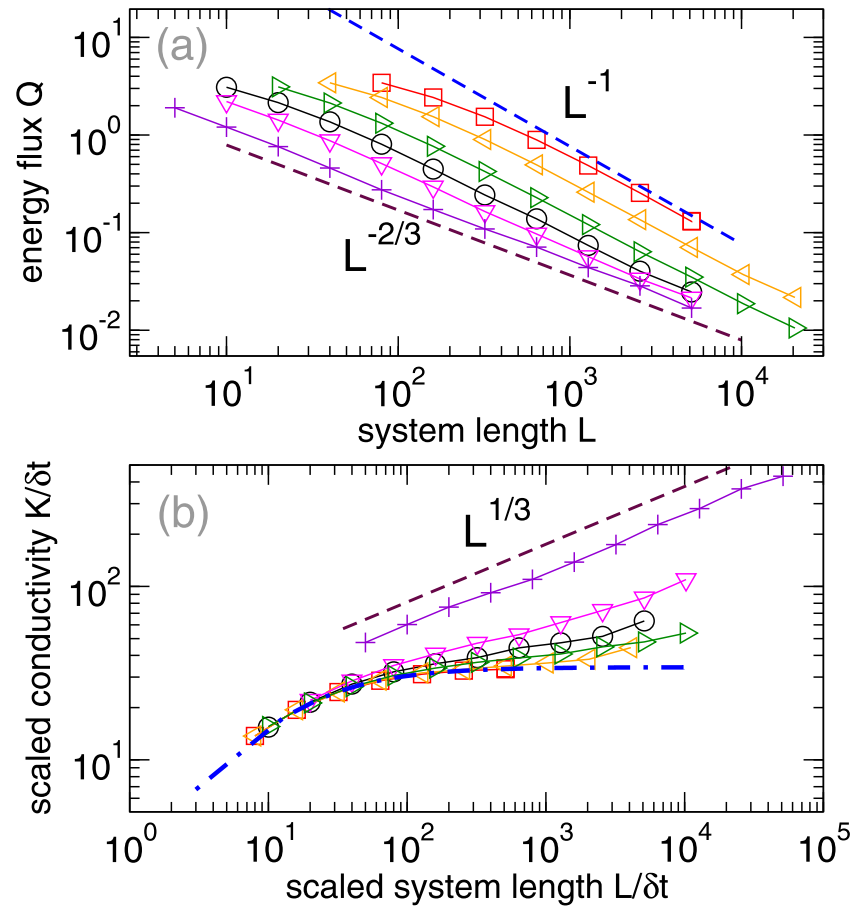

FIG. 1. Simulations of MPC fluid with density $d=5, T_{0}=4$, and $T_{L}=2$. (a) Scaling of the energy fluxes $Q$ as a function of the size $L$ for increasing collision times $\delta t=0.1,0.5,1.0,2.0,5.0$, and 10 (bottom to top); the upper and the lower dashed lines correspond to the scaling of normal and anomalous transport, respectively. (b) Scaling of the heat conductivity $K=Q L / \Delta T$ with the system size $L$; the dot-dashed blue line is a fit of the data with $\delta t=10$ with the functional form 34.2x/(12.2+x); see Eq. (56) in Ref. [3].

$x=i / L$, where $i$ is the cell index. This representation allows one to conclude also that local equilibrium is attained, since the product $T(x) n(x)$ is $x$ independent, as expected for an ideal gas (data not shown).

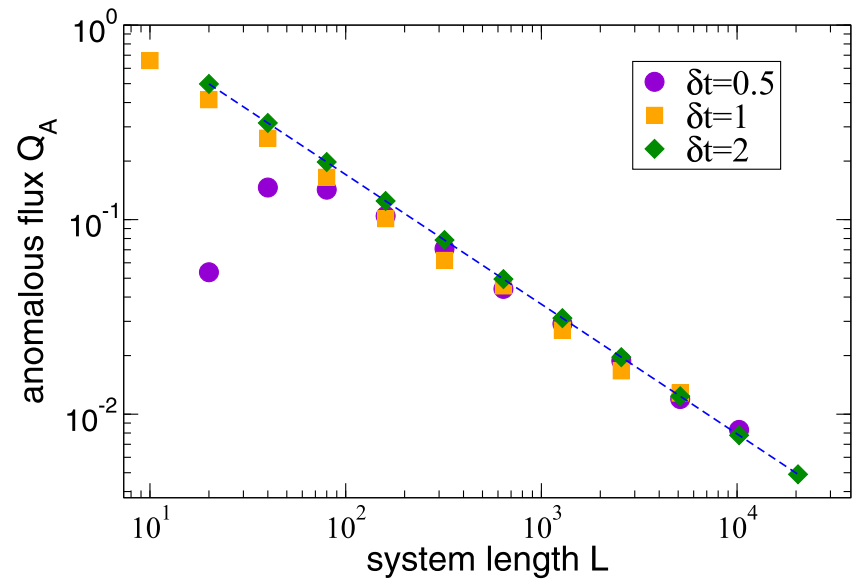

FIG. 2. Scaling of the anomalous component of the energy currents $Q_{A}$ for $d=5, T_{0}=4$, and $T_{L}=2$ and different collision times. The flux $Q_{A}$ is computed subtracting the normal component $Q_{A}$ as detailed in the text. The dashed line is a best fit of the data with $\delta t=2$, yielding a decay $Q_{A} \propto L^{-0.66(5)}$. 


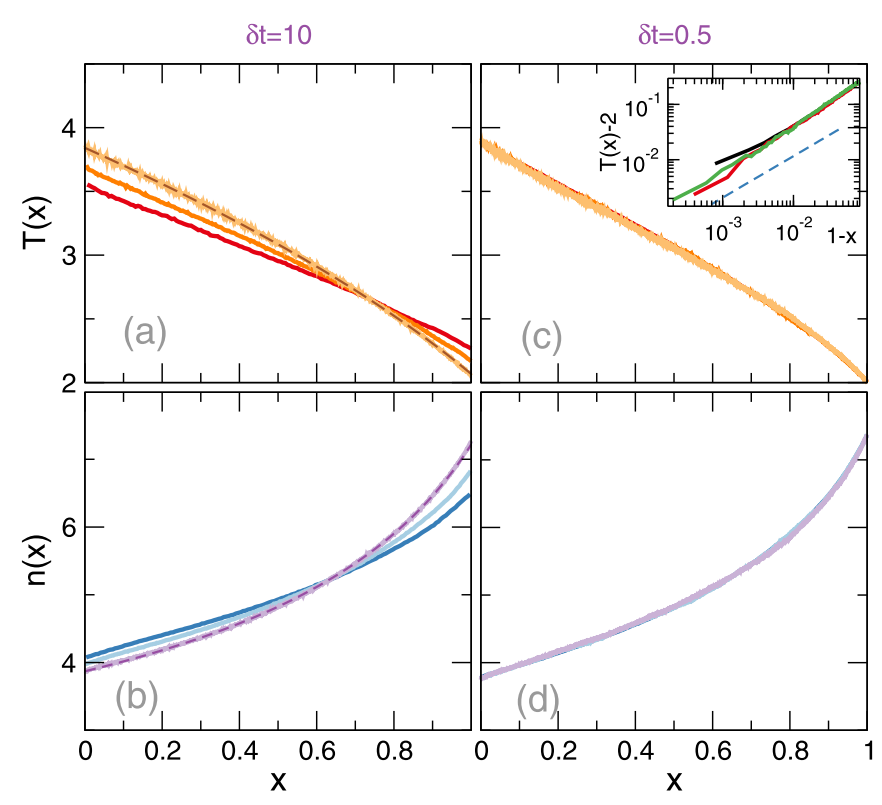

FIG. 3. Simulations of MPC fluid with density $d=5, T_{0}=4$, and $T_{L}=2$. Kinetic regime $(\delta t=10)$ : temperature (a) and density (b) profiles for $L=160,320,1280$ (bottom to top); dashed lines are fits with the function in Eq. (8) yielding $T_{0}=3.9, T_{L}=2.0$. Hydrodynamic $(\delta t=0.5)$ regime: temperature (c) and density (d) profiles for $L=640,1280,2560$; the inset in (c) shows the singularity at the right edge of the temperature profile, the dashed line corresponding to a power-law behavior $T(x) \sim(1-x)^{3 / 4}$ (see text for details).

In Fig. 3 we report these profiles for two different values of the collision time and different sizes $L$. For $L<L_{*}$ the temperature profiles are in good agreement with the prediction of Fourier's law, with thermal conductivity given by the kinetic theory, namely, $K(T) \propto T$. In fact, solving the stationary heat equation $\left[K(T) T^{\prime}\right]^{\prime}=0$ on the domain $[0, L]$ with imposed boundary temperature $T(0)=T_{0}$ and $T(1)=T_{L}$, one obtains

$$
T(x)=\left[T_{L}^{2}+\left(T_{0}^{2}-T_{L}^{2}\right) x\right]^{1 / 2} .
$$

As shown in Fig. 3(a) this functional form accounts for the measured shapes for large enough $L$, apart from the temperature jumps at the edges, that are a typical manifestation of boundary impedance effects. A similar situation was found also for the hard-point-gas (HPG) model [31,32]. Also, considerations of the same type apply to the density profiles in Fig. 3(b), in view of the relation $T(x) n(x) \approx$ const.

In the hydrodynamic regime, i.e., $L>L_{*}$, the temperature profiles exhibit instead the typical signatures of anomalous transport, signaled by singularities at the boundaries [see Figs. 3(c) and 3(d)]. For instance, the inset in Fig. 3(c) shows that $T(x) \sim(1-x)^{\mu}$ for $x \rightarrow 1$. This property is traced back to the fact that steady-state profiles should satisfy a fractional heat equation [33]. The parameter $\mu$ has been termed the meniscus exponent $\mu$, as it described the characteristic curvature close to the edges. In the simulations here, $\mu \approx 3 / 4$, a value already found for other models with reflecting boundary conditions $[33,34]$.

\section{GENERAL THERMAL WALLS}

Having in mind the possibility of simulating the injection of hot and/or cold particles into a fusion plasma by the MPC protocol, in this section we describe how one can cope with this task by introducing suitable and more general boundary conditions. Within the adopted thermal-wall scheme this can be achieved by changing the distributions of reinjected particles at one boundary of the space region. For instance, it can be assumed that the distribution $p_{0}$ on the left boundary is replaced by a function of the general form [35]

$$
p_{0}=v H(v) .
$$

By imposing the condition $\int_{0}^{\infty} v H(v) d v=1$, we ensure that the net flux of particles vanishes [35]. This distribution can be characterized by two parameters, namely, the typical average velocity of the injected particles

$$
v_{0}=\int_{0}^{\infty} v^{2} H(v) d v
$$

and the typical velocity variance

$$
(\Delta v)^{2}=\int_{0}^{\infty} v^{3} H(v) d v-v_{0}^{2},
$$

which measures the source spread [36].

As a specific example, here we consider a shifted Maxwellian distribution

$$
H(v)=H_{0}(V) e^{-(v-V)^{2} / 2 T_{0}},
$$

parametrized by the velocity $V$, where $H_{0}$ is a suitable normalization factor. Shifted Maxwellian sources are implemented in kinetic models to account for drift speed of plasmas [37]. Manifestly, both $v_{0}$ and $\Delta v$ are functions of $V$ and $T_{0}$. In the following simulations, we generate random velocities drawn from (9) and use $V$ and $T_{0}$ as control parameters, reporting also the corresponding values of $v_{0}$ and $\Delta v$ for comparison [38].

In Fig. 4 we present the results of MPC simulations for different values of $\delta t$ and $V$. We first of all note that, even setting equal temperatures at the thermal walls, i.e., $T_{0}=T_{L}$, the system is out of equilibrium and there is a net flux of energy and particles. For what concerns the scaling of the flux with the size $L$, the effect of these thermostats is pretty similar to the standard case discussed in the previous section. As shown in Fig. 4(a), in the case of relatively large collisionality the anomalous scaling [Eq. (3)] is attained. For weak collisionality [Fig. 4(b)] the diffusive scaling is found in the considered range. Although not observed in the data, we expect the same crossover described above beyond the hydrodynamic scale. In both cases, as expected, one observes that the magnitude of the current increases upon increasing the speed of the injected particles. The above results are not obvious and indicate that regardless of the nature of the baths the overall scenario is mostly dictated by bulk properties.

To better appreciate the effect of this kind of thermal wall, we measured the position-dependent steady-state distributions $f(x, v)$ in phase space for a moderate collisional parameter $\delta t=10$ (see Fig. 5). We consider two cases:

(1) The typical entry speed $v_{0}$ is of the same order of the thermal velocity $v_{0} \sim \Delta v=\sqrt{T_{0}}$ [Figs. 5(a) and 5(c)]. In 


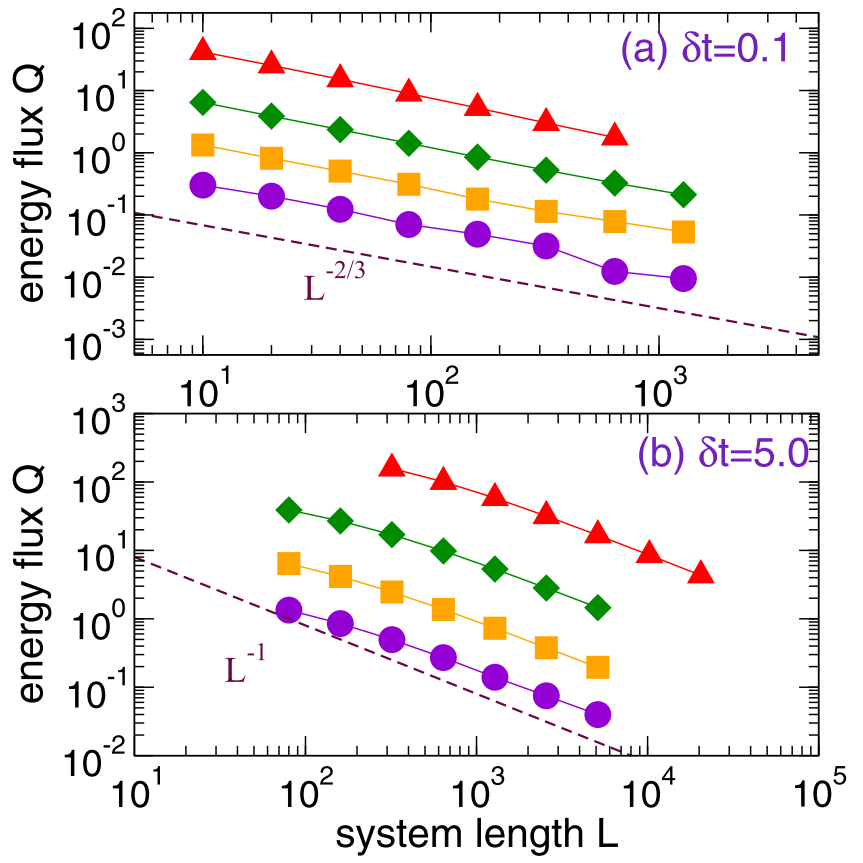

FIG. 4. Simulations with thermal wall given by Eq. (9) on the left-hand boundary for different values of the parameter $V$, with $T_{0}=$ $T_{L}=3, d=5$, and (a) $\delta t=0.1$ or (b) $\delta t=5 . V=1,2,4$, and 8 from bottom to top. The dashed lines denote the asymptotic decay corresponding to normal and anomalous transport.

this case the situation is very similar to the standard thermal bath: Temperature and density gradients are formed, while velocity distributions are Maxwellian even relatively close to the left boundary, thus indicating that local equilibrium has been achieved pretty fast.

(2) Fast, i.e., "cold," particles are injected; that is, the typical entry speed $v_{0}$ is larger than $\Delta v$ [Figs. 5(b) and 5(d)]. In this case one observes that the beam of particles entering from the left wall propagates, while experiencing the effect of the interactions with the cloud of almost-thermalized particles only in the center of the space region. As a result, the velocity distributions are strongly non-Maxwellian and remain double humped in the first half of the space region.

Adopting a kinetic point of view, we expect that the typical size of the region in which the fluid is not in local equilibrium would be of the order of mean free path $\ell$. For the simulations above, we checked that upon increasing $L$ such a region remains indeed finite. On the other hand, in anomalously conducting systems, a lack of thermal equilibrium close to the sources is found [39], and we cannot exclude that the same occurs here for larger systems.

To conclude this section, we briefly discuss the dependence of fluxes and profiles on the thermal-wall parameters. In Fig. 6(a) we plot the contour levels of the energy flux $Q$ as a function of $V$ and $T_{0}$ keeping the other parameters fixed. As remarked above, $Q$ increases with $V$ as intuitively expected. However, there is an interesting feature occurring for small enough $T_{0}$ and $V$. For $T_{0}<T_{L}$ and small $V$, injected particles are still relatively slow and thermalize quickly, so energy mostly flows from right to left, $Q<0$. The corresponding distributions and profiles $T(x)$ and $n(x)$ are shown in Figs. 6(b) and $6(\mathrm{~d})$, where it is seen that $T$ is decreasing. However, increasing the speed of the injected particles leads to a sign reversal of the current, $Q>0$, and of the associated gradients
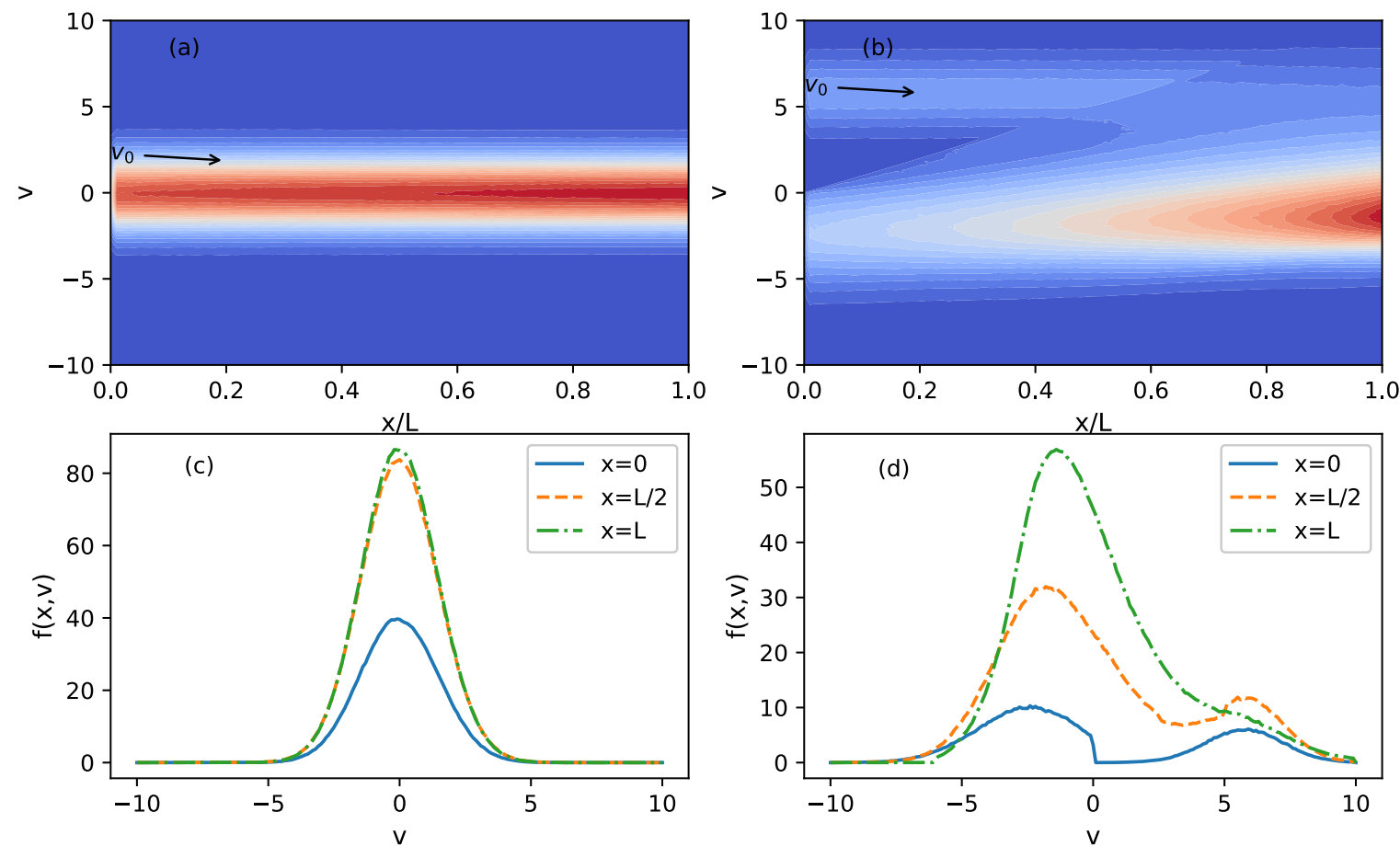

FIG. 5. Simulations with thermal wall defined by Eq. (9). (a) and (b) Particle distributions $f(x, v)$ in the steady state, and (c) and (d) their cuts at different positions. The arrows indicate the typical injection velocity $v_{0} ; T_{0}=2, T_{L}=2, L=160$, density $d=5$, and $\delta t=10$. (a) and (c) Injection of slow particles $(V=0.5)$, with $v_{0}=1.87$ and $\Delta v=1.0$. (b) and (d) Injection of fast and cold particles $(V=4)$, with $v_{0}=5.81$ and $\Delta v=1.24$. Note that even close to the right bath, $x=L$, the velocity distribution significantly deviates from a Maxwellian. 
(a)
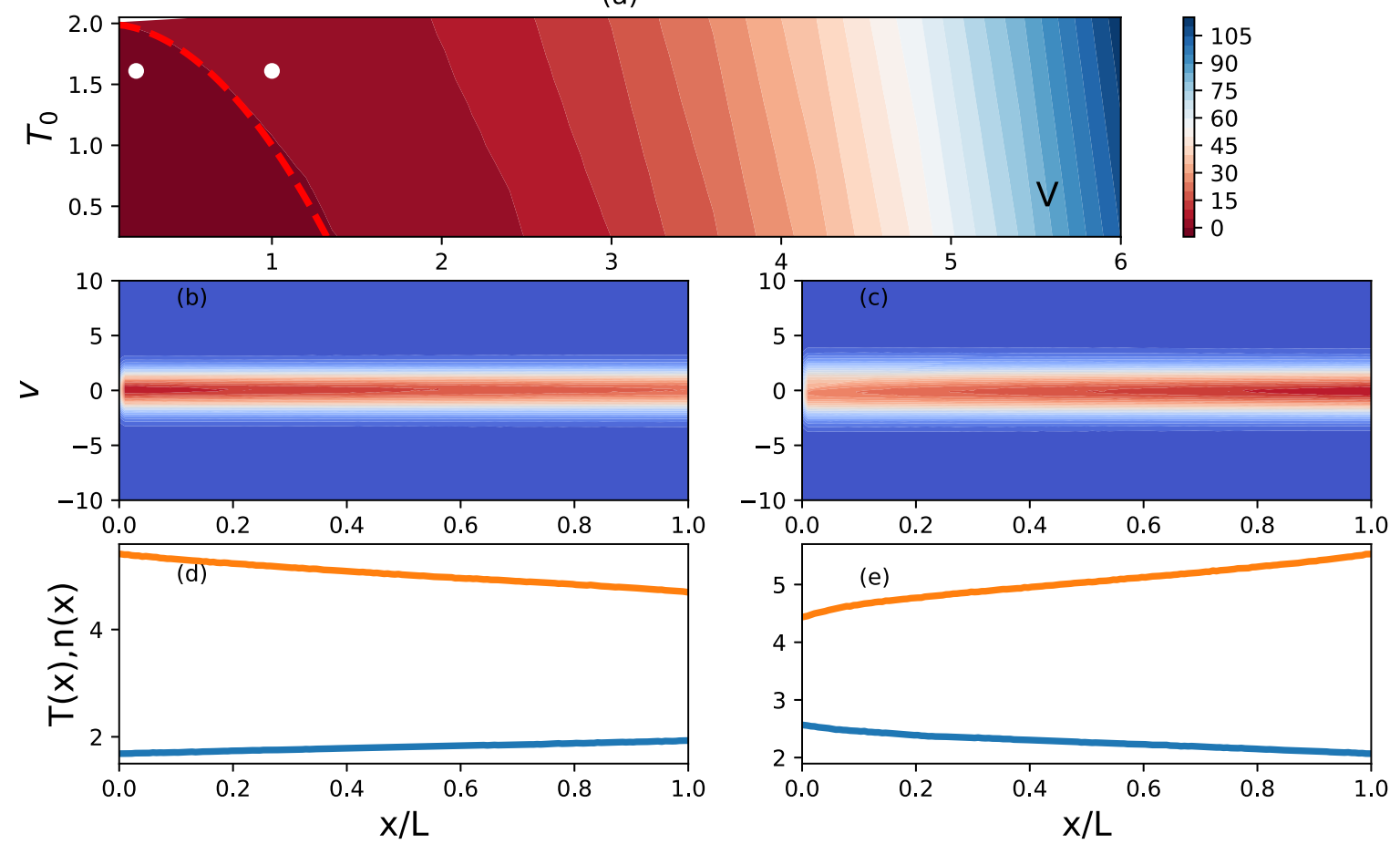

FIG. 6. Energy current reversal. (a) Contour plot of the flux $Q$ as a function of the thermal-wall parameters $V$ and $T_{0}$; simulations with other parameters fixed at $T_{L}=2, L=160, d=5$, and $\delta t=10$. The dashed red line is the curve $T_{0}=2-V^{2}$, and the energy flux is negative on the left side of it (see text for details). (b) and (c) The velocity distribution functions $f(x, v)$, and (d) and (e) temperature and density profiles (lower and upper curves, respectively). (b) and (d) are for the parameter values corresponding to $T_{0}=1.6$ and $V=0.2$, and (c) and (e) are for those corresponding to $T_{0}=1.6$ and $V=1$ [see the two white dots in (a)], showing change in the sign of the gradients.

[Figs. 6(c) and 6(e)]. A rough estimate of the conditions where this inversion takes place can be obtained by equating the kinetic temperature $T_{L}$ of the rightmost reservoir with (twice) the kinetic energies of the injected particle, which roughly gives $T_{L} \approx T_{0}+V^{2}$. As seen in Fig. 6(a) this is in agreement with the numerical data. The above estimate (dashed red line) indeed separates the two regions of the parameter plane having different signs of the energy current. On the basis of this observation, one may argue that the velocity $V$ can be used as a further control parameter of the flux direction.

\section{CONCLUSIONS}

In this paper, we have considered properties of nonequilibrium steady states and transport in a one-dimensional (1D1V) fluid in a finite-space region of length $L$ undergoing MPC dynamics and interacting with thermal walls.

For Maxwellian walls we demonstrated a crossover from a normal (kinetic) transport regime to an anomalous (hydrodynamic) one, above a characteristic size $L_{*}$. Since $L_{*}$ grows cubically with the collision time $\delta t$, the anomalous regime may hardly be detected with the sizes typically used in simulations. So we argue that a standard diffusive description will account for the observation for short enough systems. Altogether, the results nicely fit in the general framework observed for other models $[17,28,29]$. They call for a critical consideration of the usual assumptions made in kinetic theory, since memory effects and a long-time tail may play a major role in predicting heat and particle transport in low dimensions.
A natural question is whether the same scenario occurs in two-dimensional fluids. Here, the expectation is that in the hydrodynamic regime, anomalous transport would be associated with a logarithmic divergence of the conductivity with size. For the 2D MPC dynamics this is confirmed by equilibrium simulations [19]. Thus in principle a similar crossover scenario should occur, although it may be hard to observe in view of such a weak logarithmic dependence.

In the second part, we considered the more general case of thermal walls injecting particles with given average (nonthermal) velocity. We showed that the kinetic and hydrodynamic regimes are observed confirming that transport is dominated by bulk correlations in both cases. For injection of fast and relatively cold particles, short systems fail to establish local equilibrium and display non-Maxwellian distributions of velocities. We also showed that the injection velocity may be used to control current reversal.

To conclude, let us comment on the perspective of applying the model to confined plasma [40]. This requires considering the effect of the self-consistent electrostatic field, the solution of the associated Poisson equation. As already noticed in Refs. [41,42], the case of equal masses and charges in one dimension can be mapped in the so-called ding-dong model [43]. The latter consists of a one-dimensional array of identical harmonic oscillators (each one with frequency $\omega_{p}$, the plasma frequency) undergoing hard-core collisions [43]. Nonequilibrium and transport properties of such a model have been investigated in detail [41-43], and normal diffusive transport has been demonstrated, as expected since energy 
remains the only conserved quantity. One may thus argue that adding MPC dynamics will not affect significantly such a property and that the hydrodynamic regime will not be present. However, for finite systems the situation may be more subtle. Actually, the field introduces another relevant time scale in the dynamics, i.e., the inverse of the plasma frequency $\omega_{p}$, associated with the collective oscillations and Langmuir waves. This timescale should be compared with the collision frequency $1 / \delta t$. Generally speaking, if $\omega_{p} \delta t \ll 1$, energy transport will be driven mostly by collisions, and it is not a priori excluded that hydrodynamic effects may play a role over a relevant range of scales. Those issues deserve investigation.

\section{ACKNOWLEDGMENTS}

This work has been carried out within the framework of the EUROfusion Consortium and has received funding from the Euratom Research and Training Programme, 2014-2018 and 2019-2020, under Grant Agreement No. 633053. The views and opinions expressed herein do not necessarily reflect those of the European Commission. This work is part of Eurofusion Enabling Research Project ENR-MFE19.CEA-06 "Emissive divertor"; S.L. and R.L. acknowledge partial support from Project MIUR-PRIN2017 "Coarse-grained description for non-equilibrium systems and transport phenomena $(\mathrm{CO}-$ NEST),” No. 201798CZL.
[1] A. Malevanets and R. Kapral, Mesoscopic model for solvent dynamics, J. Chem. Phys. 110, 8605 (1999).

[2] R. Kapral, Multiparticle collision dynamics: Simulation of complex systems on mesoscales, in Advances in Chemical Physics, edited by S. A. Rice (Wiley, New York, 2008), Vol. 140, pp. 89-146.

[3] S. Lepri, R. Livi, and A. Politi, Thermal conduction in classical low-dimensional lattices, Phys. Rep. 377, 1 (2003).

[4] A. Dhar, Heat transport in low-dimensional systems, Adv. Phys. 57, 457 (2008).

[5] Thermal Transport in Low Dimensions: From Statistical Physics to Nanoscale Heat Transfer, edited by S. Lepri, Lecure Notes in Physics Vol. 921 (Springer-Verlag, Berlin, 2016).

[6] H. van Beijeren, Exact Results for Anomalous Transport in One-Dimensional Hamiltonian Systems, Phys. Rev. Lett. 108, 180601 (2012).

[7] H. Spohn, Nonlinear fluctuating hydrodynamics for anharmonic chains, J. Stat. Phys. 154, 1191 (2014).

[8] O. Narayan and S. Ramaswamy, Anomalous Heat Conduction in One-Dimensional Momentum-Conserving Systems, Phys. Rev. Lett. 89, 200601 (2002).

[9] L. Delfini, S. Lepri, R. Livi, and A. Politi, Self-consistent modecoupling approach to one-dimensional heat transport, Phys. Rev. E 73, 060201(R) (2006).

[10] A. Pereverzev, Fermi-Pasta-Ulam $\beta$ lattice: Peierls equation and anomalous heat conductivity, Phys. Rev. E 68, 056124 (2003).

[11] B. Nickel, The solution to the 4-phonon Boltzmann equation for a 1D chain in a thermal gradient, J. Phys. A: Math. Theor. 40, 1219 (2007).

[12] J. Lukkarinen and H. Spohn, Anomalous energy transport in the FPU- $\beta$ chain, Commun. Pure Appl. Math. 61, 1753 (2008).

[13] S. Lepri, R. Livi, and A. Politi, Universality of anomalous one-dimensional heat conductivity, Phys. Rev. E 68, 067102 (2003).

[14] L. Wang and T. Wang, Power-law divergent heat conductivity in one-dimensional momentum-conserving nonlinear lattices, EPL 93, 54002 (2011).

[15] S. Liu, P. Hänggi, N. Li, J. Ren, and B. Li, Anomalous Heat Diffusion, Phys. Rev. Lett. 112, 040601 (2014).

[16] G. Benenti, S. Lepri, and R. Livi, Anomalous heat transport in classical many-body systems: Overview and perspectives, Front. Phys. 8, 292 (2020).
[17] S. Lepri, R. Livi, and A. Politi, Too Close to Integrable: Crossover from Normal to Anomalous Heat Diffusion, Phys. Rev. Lett. 125, 040604 (2020).

[18] P. Di Cintio, R. Livi, H. Bufferand, G. Ciraolo, S. Lepri, and M. J. Straka, Anomalous dynamical scaling in anharmonic chains and plasma models with multiparticle collisions, Phys. Rev. E 92, 062108 (2015).

[19] P. Di Cintio, R. Livi, S. Lepri, and G. Ciraolo, Multiparticle collision simulations of two-dimensional one-component plasmas: Anomalous transport and dimensional crossovers, Phys. Rev. E 95, 043203 (2017).

[20] P. Stangeby, The Plasma Boundary of Magnetic Fusion Devices, Plasma Physics Series (Institute of Physics, Philadelphia, 2000), Chap. 26.

[21] S. Krasheninnikov, A. Smolyakov, and A. Kukushkin, On the Edge of Magnetic Fusion Devices, Springer Series in Plasma Science and Technology (Springer, New York, 2020).

[22] D. J. Bond, Approximate calculation of the thermal conductivity of a plasma with an arbitrary temperature gradient, J. Phys. D: Appl. Phys. 14, L43 (1981).

[23] H. Bufferand, G. Ciraolo, P. Ghendrih, P. Tamain, F. Bagnoli, S. Lepri, and R. Livi, One-dimensional particle models for heat transfer analysis, J. Phys.: Conf. Ser. 260, 012005 (2010).

[24] H. Bufferand, G. Ciraolo, Ph. Ghendrih, S. Lepri, and R. Livi, Particle model for nonlocal heat transport in fusion plasmas, Phys. Rev. E 87, 023102 (2013).

[25] M. Kocan, R. A. Pitts, G. Arnoux, I. Balboa, P. C. De Vries, R. Dejarnac, I. Furno, R. J. Goldston, Y. Gribov, J. Horacek, M. Komm, B. Labit, B. LaBombard, C. J. Lasnier, R. Mitteau, F. Nespoli, D. Pace, R. Panek, P. C. Stangeby, J. L. Terry et al., Impact of a narrow limiter SOL heat flux channel on the ITER first wall panel shaping, Nucl. Fusion 55, 033019 (2015).

[26] J. P. Gunn, S. Carpentier-Chouchana, F. Escourbiac, T. Hirai, S. Panayotis, R. A. Pitts, Y. Corre, R. Dejarnac, M. Firdaouss, M. Kočan, M. Komm, A. Kukushkin, P. Languille, M. Missirlian, W. Zhao, G. Zhong et al., Surface heat loads on the ITER divertor vertical targets, Nucl. Fusion 57, 046025 (2017).

[27] R. Tehver, F. Toigo, J. Koplik, and J. R. Banavar, Thermal walls in computer simulations, Phys. Rev. E 57, R17 (1998).

[28] H. Zhao and W.-g. Wang, Fourier heat conduction as a strong kinetic effect in one-dimensional hard-core gases, Phys. Rev. E 97, 010103(R) (2018). 
[29] A. Miron, J. Cividini, A. Kundu, and D. Mukamel, Derivation of fluctuating hydrodynamics and crossover from diffusive to anomalous transport in a hard-particle gas, Phys. Rev. E 99, 012124 (2019).

[30] K. Aoki and D. Kusnezov, Fermi-Pasta-Ulam $\beta$ Model: Boundary Jumps, Fourier's Law, and Scaling, Phys. Rev. Lett. 86, 4029 (2001).

[31] A. Dhar, Heat Conduction in a One-Dimensional Gas of Elastically Colliding Particles of Unequal Masses, Phys. Rev. Lett. 86, 3554 (2001).

[32] P. Grassberger, W. Nadler, and L. Yang, Heat Conduction and Entropy Production in a One-Dimensional Hard-Particle Gas, Phys. Rev. Lett. 89, 180601 (2002).

[33] S. Lepri and A. Politi, Density profiles in open superdiffusive systems, Phys. Rev. E 83, 030107(R) (2011).

[34] A. Kundu, C. Bernardin, K. Saito, A. Kundu, and A. Dhar, Fractional equation description of an open anomalous heat conduction set-up, J. Stat. Mech.: Theory Exp. (2019) 013205.

[35] N. Ianiro and J. L. Lebowitz, Stationary nonequilibrium solutions of model Boltzmann equation, Found. Phys. 15, 531 (1985).

[36] Compare with the case of a standard Maxwellian bath with $V=0$ and $H(v)=\beta e^{-\beta v^{2} / 2}$; using the integrals $v_{0}=$ $\int_{0}^{\infty} v^{2} H(v) d v=\sqrt{\pi / 2 \beta}$ and $\int_{0}^{\infty} v^{3} H(v) d v=2 / \beta$, one finds
$v_{T}=\sqrt{0.429 . . / \beta}$. Thus $v_{0}$ and $\Delta v$ are both proportional to $\sqrt{T}$ and cannot be changed independently.

[37] J. P. Gunn, Kinetic investigation of a collisionless scrape-off layer with a source of poloidal momentum, J. Nucl. Mater. 337, 310 (2005).

[38] In order to generate random deviates with the above distribution, one can take $v=\sqrt{X^{2}+Y^{2}}$, where $X$ and $Y$ are independent and identically distributed (iid) Gaussian variables with nonzero average $A$. In practice, $A$ is fixed to some preassigned value, and the resulting $V$ and $v_{0}$ are measured by fitting the sampled distribution [Eq. (9)].

[39] L. Delfini, S. Lepri, R. Livi, C. Mejía-Monasterio, and A. Politi, Nonequilibrium dynamics of a stochastic model of anomalous heat transport: numerical analysis, J. Phys. A: Math. Theor. 43, 145001 (2010).

[40] J. Dawson, One-dimensional plasma model, Phys. Fluids (1958-1988) 5, 445 (1962).

[41] M. M. Sano and K. Kitahara, Thermal conduction in a chain of colliding harmonic oscillators revisited, Phys. Rev. E 64, 056111 (2001).

[42] M. M. Sano and K. Kitahara, Kinetic theory of Dawson plasma sheet model, J. Phys. Soc. Jpn. 80, 084001 (2011).

[43] T. Prosen and M. Robnik, Energy-transport and detailed verification of Fourier heat law in a chain of colliding harmonicoscillators, J. Phys. A: Math. Gen. 25, 3449 (1992). 